\title{
Evaluación de la equidad del servicio de transporte público: El caso de Palma de Mallorca
}

\section{Assessing equity of public transport: the case of Palma (Mallorca, Illes Balears)}

\author{
Maurici Ruiz, Joana Maria Seguí Pons, Jaume Mateu Lladó \\ y Maria Rosa Martínez Reynés ${ }^{1}$
}

\begin{abstract}
RESUMEN
La planificación sostenible del transporte público requiere la consideración de la equidad territorial y social. El análisis de la equidad precisa de un profundo conocimiento del servicio así como de las características territoriales y sociales dónde se implanta. En este artículo se propone un método simplificado para el análisis de la equidad del sistema de autobuses públicos, aplicado a la ciudad de Palma. El nivel de servicio se calcula a partir del análisis territorial de la oferta, y se contrasta con la población existente y con un índice multidimensional de necesidad social de transporte público, dando lugar a la equidad horizontal y vertical, respectivamente. Posteriormente, se verifica la equidad global del servicio con el apoyo de la curva de Lorenz y del coeficiente GINI. Finalmente, se realiza un análisis de sensibilidad a través de la frecuencia de las rutas de autobús en relación a la equidad social del servicio,
\end{abstract}

1 Maurici Ruiz, director del Servicio de SIG y Teledetección,Vicerectorat d'Innovació i Transferència, Universitat de les Illes Balears, maurici.ruiz@uib.es. Joana María Seguí Pons, Catedrática, Departament de Ciències de la Terra, Universitat de les Illes Balears, joana.seguipons@uib.es. Jaume Matéu Lladó, geógrafo, Investigador contratado, Departament de Ciències de la Terra, Universitat de les Illes Balears, jaume.mateu@uib.cat. María Rosa Martínez Reynés, Universitat de les Illes Balears, fallecida el 15 de enero de 2015, descanse en paz. 
Palabras Clave: equidad del transporte; curva de Lorenz; coeficiente GINI; sistema de información geográfica; análisis multicriterio.

\begin{abstract}
The practice of sustainable transport planning must take into account the level of territorial and social equity of service. The equity analysis requires a deep understanding of the service and the territorial and social characteristics where it is implanted. We propose a simplified method to analyze the equity of the public bus system that has been used in the city of Palma de Mallorca. The bus service level was calculated from the spatial analysis of the offer and was contrasted with the population and with a multidimensional index of social need for public transportation leading to horizontal and vertical equity respectively. Next the overall equity of the service was tested with the support of the Lorenz curve and Gini coefficient. Finally, a sensitivity analysis of bus routes depending on the role they play in the equity of the service was performed.
\end{abstract}

KEY WORDS: transport equity; network analyst; Lorenz Curve; Gini coefficient; GIS transport; multicriteria analysis.

\title{
INTRODUCCIÓN
}

\section{Transporte público y equidad}

Un transporte público equilibrado implica sostenibilidad ambiental, social y económica y constituye un requisito destacado para asegurar la movilidad y la calidad de vida de los habitantes en las ciudades que disponen de este servicio. El equilibrio entre la oferta y la demanda en el transporte público supone un proceso de continua mejora en las infraestructuras -fijas y móviles-, en los servicios y en los incentivos a su uso, así como una adaptación dinámica al modelo social y económico de los usos del suelo urbano. De hecho, el modelo de transporte y movilidad constituye una de las bases de la sostenibilidad urbana que debe dar coherencia al modelo urbanístico existente. En las relaciones transportes-usos del suelo el transporte público constituye un importante instrumento de justicia social ya que puede contribuir de forma más racional al crecimiento de las ciudades haciendo posible una accesibilidad más equitativa para las posibilidades de empleo, cultura, ocio, etc. (Cocco y Silveira, 2011, p.555). Los usos del suelo y los sistemas de transporte interaccionan de forma dinámica a varias escalas y determinan la sostenibilidad ambiental y social del entorno urbano (Litman, 2014).

La sostenibilidad social del transporte hace referencia a la capacidad de proporcionar a todos los miembros de la sociedad los mismos niveles de ac- 
ceso a diferentes oportunidades y servicios (Casas, 2007). El transporte público proporciona acceso a materias, servicios y actividades, ayuda a la población a desplazarse a su lugar de residencia o de trabajo, a comprar, estudiar ir a un hospital o acceder a cualquier otro servicio urbano por lo que se nos aparece como una variable imprescindible a la hora de abordar las cuestiones de oportunidad y equidad (Litman, 2002). La falta o dificultad de acceso al sistema de transporte público puede considerarse, entre otras, una importante causa de exclusión social.

En este punto resulta obligado diferenciar el concepto de sostenibilidad social del transporte y éste como instrumento de justicia social, anteriormente desarrollados, de otras acepciones del concepto de «sostenibilidad», tan sólo referido al medio ambiente en sus inicios, o del desarrollo sostenible, o del propio concepto de justicia ambiental (Pedersen, 2010). Éste último, aunque difícil de definir y con múltiples acepciones, de ahí su éxito, proporciona el marco para la aplicación de leyes y normativas relativas al desarrollo sostenible.

Retomando el concepto de equidad, en él intervienen aspectos económicos y sociales pero también relativos a las infraestructuras y al servicio, por lo que este enfoque multidimensional se está integrando de forma progresiva en los estudios de movilidad y transporte. Al respecto, en la planificación del transporte, se distinguen dos tipos de equidad territorial del servicio, la horizontal y la vertical. La horizontal tiene que ver con la justicia espacial en el mantenimiento de una oferta equilibrada de transporte público, adecuada a las necesidades de desplazamiento de todos los individuos y que permita, en primer lugar, el acceso a la educación, al empleo y a los servicios y, seguidamente, al desarrollo de actividades recreativas y sociales. La equidad vertical hace referencia al nivel de adaptación de la oferta de transporte a las necesidades exclusivas de determinados grupos de población. Así, los que manifiestan una mayor necesidad de transporte público urbano son los colectivos con menor nivel de renta, mayor grado de dependencia, entre otras razones por cuestiones de edad, y menores recursos personales. Desde este enfoque, la equidad del transporte puede ser considerada como un bien de interés social o incluso un derecho, pues en la praxis de la planificación del transporte público, resulta obligado considerar la equidad social como propiedad primordial para garantizar el acceso y uso equilibrado de la población a dicho servicio (Hernández et al., 2011).

La planificación del servicio de autobús requiere el desarrollo de un conjunto de tareas entre las que se incluyen: el análisis de la demanda, la selección de rutas y paradas, el establecimiento de frecuencias horarias, la selección de vehículos, etc. (Cipriani, Gori et al., 2012; Farahani, Miandoab- 
chi et al., 2013). Cada uno de dichos aspectos puede ser objeto de un enfoque social, sin embargo, en la mayoría de los casos, los intereses económicos y la rentabilidad de los servicios suelen priorizarse sobre los factores sociales.

Existen diversas metodologías para el análisis de la equidad espacial del transporte público, aunque son escasos los métodos efectivos de optimización del transporte en base al mantenimiento de la equidad social.

En este trabajo se incide en el análisis de la sostenibilidad social del modelo de transporte, proponiendo la adaptación de la metodología integrada propuesta por Currie (Currie, 2010; Delbosc y Currie, 2011) al ámbito de una ciudad mediterránea de tamaño medio. La metodología se enriquece incorporando un modelo multicriterio basado en un proceso analítico jerárquico (AHP, por sus siglas en inglés) para el análisis de la demanda de transporte y el análisis de la sensibilidad de las líneas de autobuses.

\section{Área de estudio}

El ámbito geográfico de estudio es la ciudad de Palma (Mallorca, Islas Baleares) (figura 1). La planificación y gestión del transporte público compete a la Empresa Municipal de Transporte (EMT, 2014) dependiente del Ayuntamiento de Palma. La oferta de transporte público en superficie -Palma dispone de dos líneas de metro, una de reciente construcción (2007) y otra procedente de la línea de ferrocarril subterránea- se realiza a través de 31 rutas de autobús, 28 diurnas, 1 nocturna y dos turísticas, que atraviesan los distintos barrios de la ciudad recorriendo un total de 822, 5 kilómetros, con un total de 957 paradas distribuidas en un municipio de 19.535 hectáreas (figura 2). El servicio de autobús de la ciudad pretende cubrir las necesidades de una población de 421.708 residentes (2013) (IBESTAT, 2014) distribuidos en 88 barrios. El 87\% de la población, según el PMUS (Ajuntament de Palma, 2014), reside a menos de 350 metros de una parada por la que, de media, circula un autobús en día laborable cada 15 minutos. En 2013 se transportaron 38.028.160 viajeros, con una media diaria de 104.187 viajes para el conjunto de la ciudad, demanda concentrada en menos de la mitad de las líneas que aglutinan más del 85\% de los pasajeros (EMT, 2014). Al ser Palma la capital de las Baleares cuenta, además, con una población flotante procedente del resto de municipios de las Islas, sobre todo de Mallorca. Por su marcado carácter turístico -que se refleja en la dotación de 42.791 plazas turísticas (Agència de Turisme de les Illes Balears, 2013) - los efectivos demográficos aumentan sustancialmente en los meses de temporada alta. Palma ve incrementada su población en una media diaria de 
22.000 personas entre los meses de abril y octubre, esto es, en un 5\% de su población permanente. Esta situación se agudiza entre junio y septiembre y alcanza su máximo en el mes de agosto cuando la presión humana aumenta en un 10\%, con 39.697 personas diarias más en la ciudad (Ajuntament de Palma, 2013, pág. 17, 18). El municipio de Palma recibió en 2011 un total de 1.842.747 turistas, con un promedio de un poco más de cuatro días de estancia, alojados en los hoteles urbanos o situados en la costa en los barrios del Arenal y Cala Major. Mallorca recibió, en el mismo año, casi nueve millones de visitantes y las Islas Baleares, algo más de doce millones. En los últimos tres años estas cifras se han incrementado ligeramente.

El sistema viario de la ciudad de Palma está estructurado a partir de una red de morfología radiocéntrica, en torno a su casco histórico, con dos anillos perimetrales formados por las Avenidas, primer cinturón de ronda una vez derruidas las murallas, y la Vía de Cintura en el exterior de la ciudad compacta. Éstos condicionan el desarrollo de una circulación perimetral en la que convergen diversos ejes de penetración y de salida del núcleo.

\section{FIGURA 1}

\section{LOCALIZACIÓN DE LA CIUDAD DE PALMA Y DIVISIÓN EN BARRIOS.}

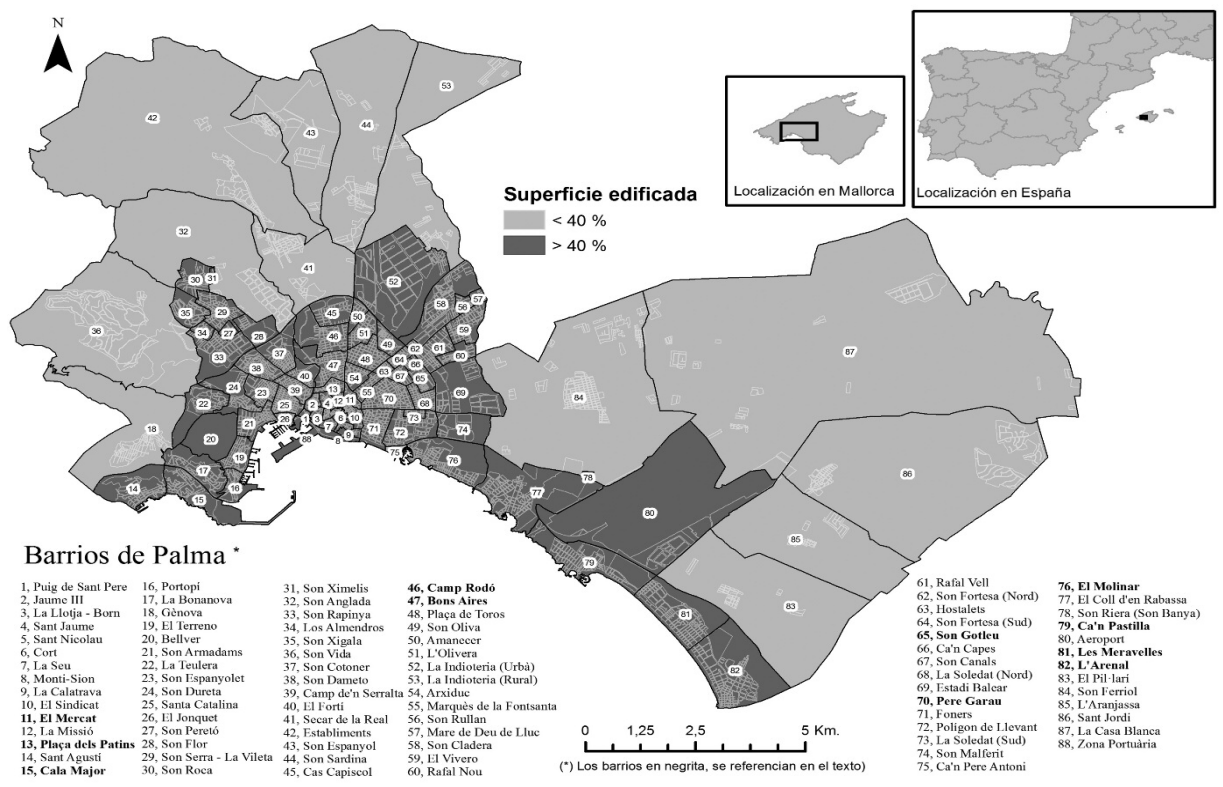

Fuente: Base cartográfica Cartociudad (Instituto Geográfico Nacional, 2014). 
El uso del transporte público es mayoritario en colectivos sociales con bajo nivel de ingresos, mujeres, estudiantes, población dependiente (personas mayores y menores) y turistas.

FIGURA 2

RUTAS DE LAS PRINCIPALES LÍNEAS DE BUS EN PALMA.

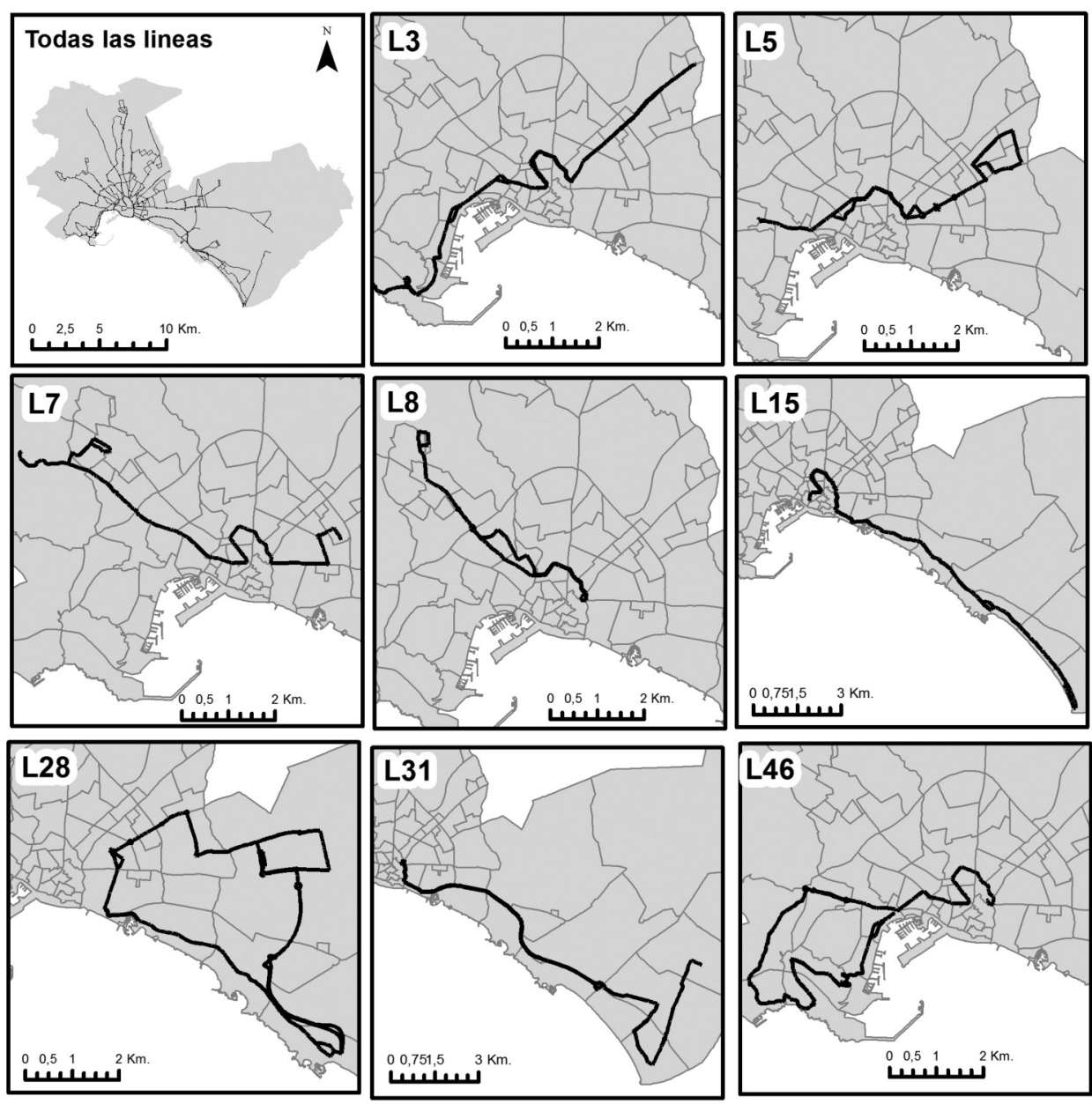

Fuente: Empresa Municipal de Transportes de Palma (EMT, 2014). 
El objetivo principal del presente trabajo radica, en la identificación de los barrios que presentan mayores desequilibrios entre la población residente y el transporte público del que disponen, debido a la falta de correspondencia entre sus necesidades demográficas y el nivel de servicio de transporte, valorado, a través de la superficie servida por las líneas, las paradas, y las frecuencias. Como objetivos secundarios, identificar las variables que mayor papel desempeñan en la consecución de la equidad vertical del transporte; otro, más bien de carácter propositivo, estriba en apuntar medidas de mejora del servicio, a través de la identificación de las líneas de transporte más significativas en cada una de las unidades de análisis. Se pretende igualmente, la implementación de una metodología exportable al análisis en otras ciudades, con otros servicios de transporte.

\section{FUENTES Y MÉTODOS}

El estudio de la equidad social del transporte público en autobús ha constado de las siguientes fases: análisis de la oferta del servicio, análisis de la demanda potencial y análisis del impacto (sensibilidad) de las frecuencias de la líneas de autobús en relación a la equidad del servicio.

\section{Análisis de la oferta del servicio de transporte público en autobús}

Una de las metodologías de planificación y evaluación del servicio de transporte más eficiente es la utilizada por G. Currie (Currie, 2010; Delbosc y Currie, 2011; Huang, 2003). Su aplicación hace posible analizar la oferta del servicio de transporte público contando únicamente con información de las paradas de autobús, las frecuencias horarias de las líneas y la superficie de los barrios que atraviesan. A pesar de que posee importantes limitaciones y falta de exhaustividad en el análisis pormenorizado del servicio, la consideramos muy adecuada para disponer de una visión global de la distribución geográfica de la oferta de transporte, pudiendo llegar a proporcionar información de gran utilidad para el desarrollo de tareas de análisis exploratorio y gestión estratégica del servicio.

La calidad en los procesos de delimitación de las áreas cubiertas por el transporte público y la estimación de la población servida constituye un tema de estudio recurrente en el ámbito científico (Moreno y Prieto, 2004) y está demostrado que resulta común cometer errores de sobreestimación (Gutiérrez 
y García-Palomares, 2008). En general, el acceso de la población a las paradas de transporte público confirma la existencia de funciones de disminución del número de usuarios en relación a la distancia que podrían influir negativamente en los resultados de los modelos (Biba, Curtin et al., 2010; García-Palomares, Gutierrez et al., 2013).

La organización territorial de la ciudad de Palma se establece en base a la división en zonas estadísticas, denominadas barrios, que fueron definidas por criterios históricos, por lo que su superficie urbanizada y peso demográfico son variables. En la mayoría de los barrios la población oscila entre las 1.000 y las 5.000 personas. Sin embargo, nueve de ellos cuentan con más de 10.000 habitantes, mientras que algunos no superan los 1.000 .

FIGURA 3

RELACIÓN ENTRE LA POBLACIÓN Y LA SUPERFICIE DE LOS BARRIOS. DE PALMA

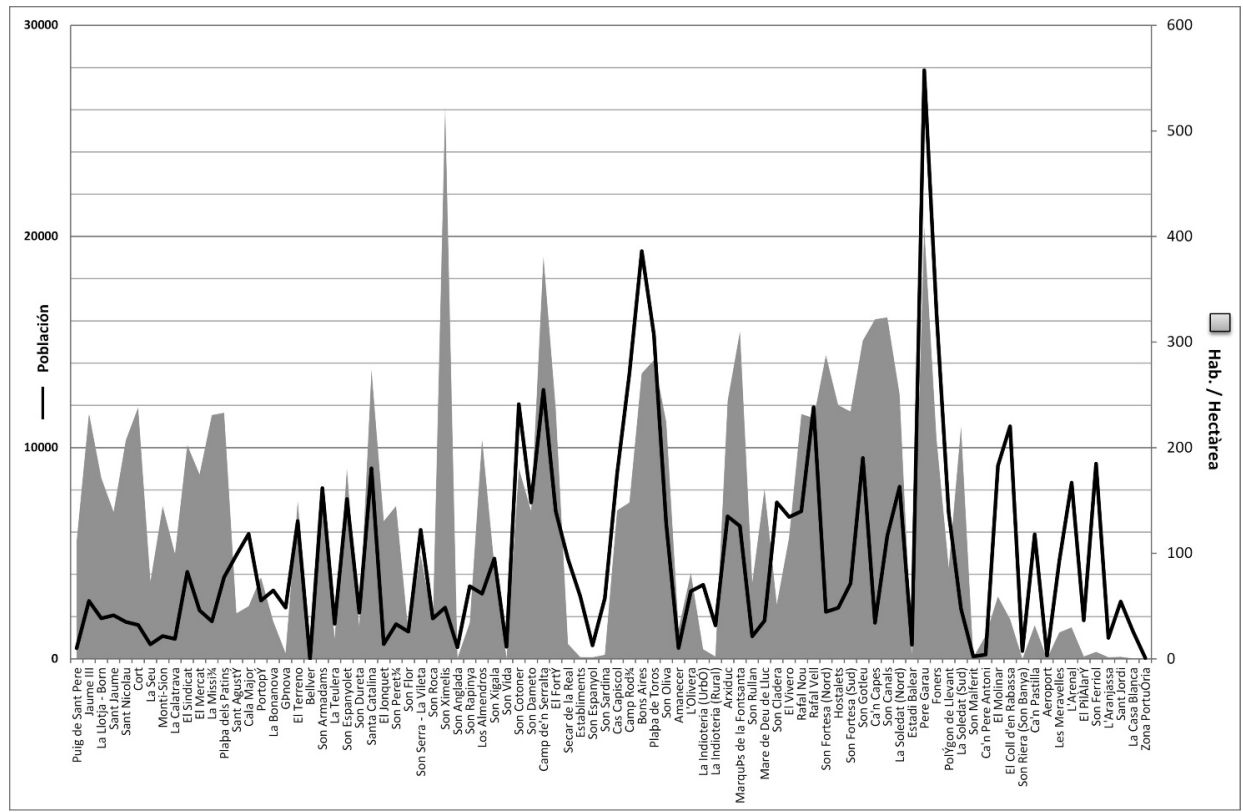

Fuente: Padrón municipal de habitantes de Palma 2012. 
Con objeto de mejorar la calidad de los resultados del análisis realizado, se ha creído conveniente seleccionar los barrios que poseen un tejido urbano más continuo y de esa forma evitar posibles errores en los cálculos derivados de la dispersión de la población. A tal efecto, a partir de la cartografía de manzanas proporcionada por el proyecto "Cartociudad" del Instituto Geográfico Nacional (IGN, 2014), se ha calculado la zona construida de cada barrio y se ha porcentualizado con su superficie total. De esa forma se seleccionan un total de 73 barrios o unidades de análisis cuya zona edificada supera el $40 \%$ del total de la superficie del barrio (figura 1).

El análisis de la oferta de servicio de autobús, expresada a través del Índice de nivel de Servicio, se realizó a través de las siguientes fases: georeferenciación de las paradas, cálculo del nivel de servicio a partir del número de autobuses por parada y evaluación del nivel de servicio de autobús por barrio. A nivel técnico se hizo uso del programa informático ArcGIS vers. 10.2 (๔) ESRI) combinado con la hoja de cálculo Excel vers. 13. (@ Microsoft).

Georeferenciación de las paradas. A partir de la información suministrada por la Empresa Municipal de Transportes de Palma se ha realizado la georreferenciación de 959 paradas de autobús correspondientes a 31 líneas.

Cálculo del nivel de servicio a partir del número de autobuses por parada. Tomando en consideración la frecuencia temporal de las líneas de bus, se obtiene el número de servicios que ofrece cada parada para un periodo de 12 horas en días laborables.

Evaluación del nivel de servicio de autobús por barrio (figuras 4 y 5 ). Se ha generado un área de influencia concéntrica de 300 metros desde cada parada. Posteriormente, se ha asignado el número de buses/día de cada parada a su correspondiente área de influencia. A continuación se han superpuesto los límites de los barrios sobre las áreas de influencia de cada parada. De esta forma, cada barrio contiene un conjunto de áreas de influencia correspondientes a distintas paradas. Finalmente, se pondera el porcentaje de barrio cubierto por cada parada con el número de buses. El valor final del índice se normaliza en una escala de 0 a 1 con objeto de poder ser combinado con los índices de demanda potencial. 


\section{FIGURA 4 \\ CÁLCULO DEL ÍNDICE DE NIVEL DE SERVICIO \\ DE TRANSPORTE PÚBLICO}

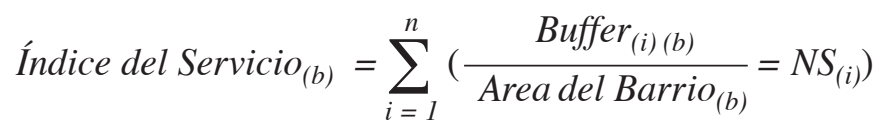

b: Barrio; i: número parada; n: número total de paradas; NS: Nivel de Servicio de la parada ( $\mathrm{n}^{\mathrm{o}}$ buses en $12 \mathrm{~h}$. de día laborable) Buffer: Superficie de la intersección del área de influencia de $300 \mathrm{~m}$. desde la parada (i) y el barrio (b)

Fuente: (Currie, 2010).

FIGURA 5

NIVEL DE SERVICIO DE TRANSPORTE PÚBLICO. PALMA. ( $\mathrm{N}^{\circ}$ DE AUTOBUSES EN 12 HORAS/DÍA LABORABLE)

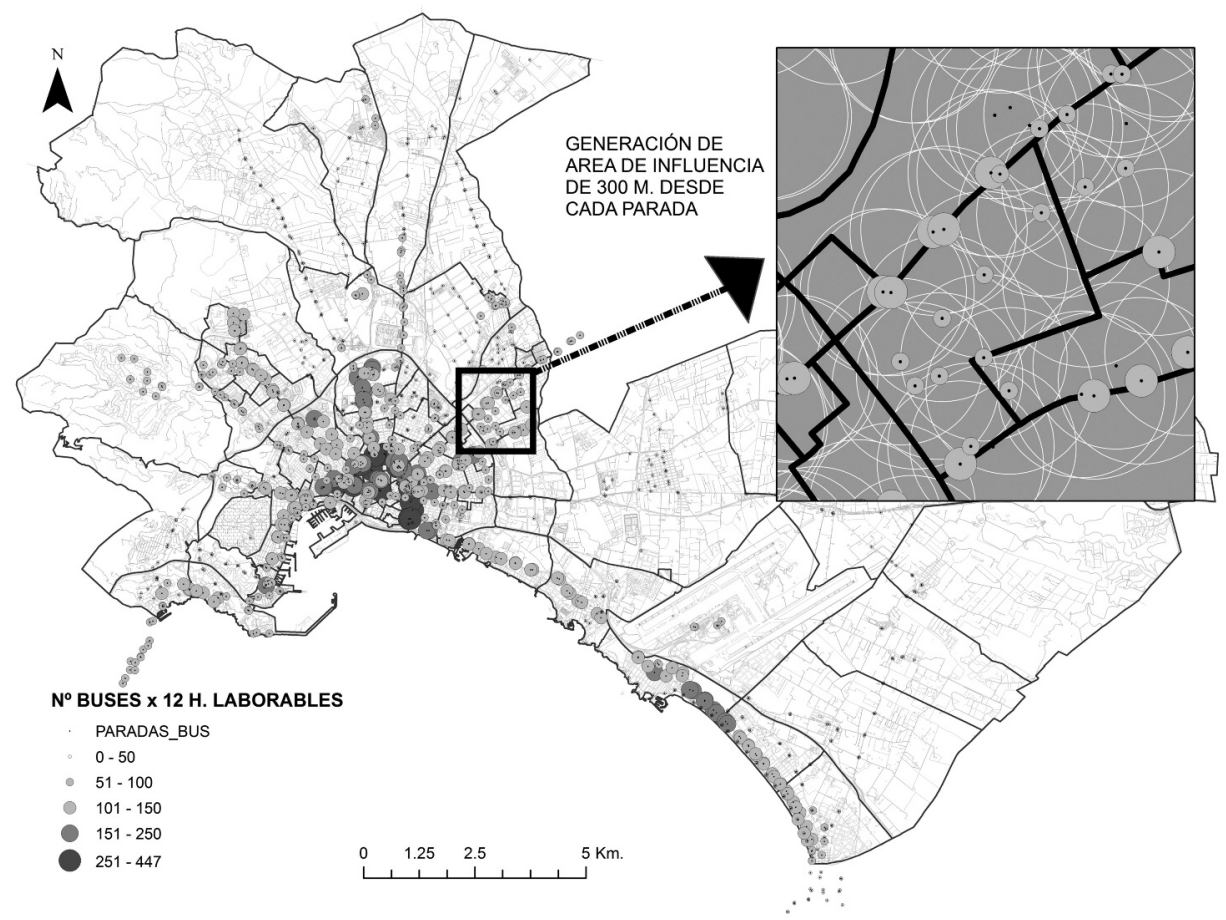

Fuente: Empresa Municipal de Transportes de Palma (EMT, 2014).

Estudios Geográficos, Vol. LXXVII, 281, pp. 619-646, julio-diciembre 2016 ISSN: 0014-1496, eISSN: 1988-8546, doi: 10.3989/estgeogr.201621 


\section{Estimación de la demanda potencial}

La predicción de la demanda de transporte es una tarea necesaria en la planificación de infraestructuras y de servicios (Ortuzar, J., 2003); (Souche, 2010). En nuestro caso, calculamos dos indicadores de la demanda de transporte. Para la evaluación de la equidad horizontal se hará uso de la densidad de población por barrio mientras que para el análisis de la equidad vertical se calculará un indicador social de la demanda de tipo cualitativo al que denominamos Índice de Necesidad Social de Transporte Público (INSTP).

TABLA 1

VARIABLES SOCIOECONÓMICAS CARACTERIZADORAS DE LOS BARRIOS DE PALMA

\begin{tabular}{|c|c|c|c|}
\hline $\mathrm{Vl}$ & EXTENSION (Km2) & V14 & No LATINOAMERICANOS \\
\hline $\mathrm{V} 2$ & $\mathrm{~N}^{\circ}$ PERSONAS & V15 & No ASIÁTICOS \\
\hline V3 & No PERSONAS / HECTAREA & V16 & $\mathrm{N}^{\circ}$ AFRICANOS \\
\hline V4 & $\mathrm{N}^{\circ}$ HOMBRES & V17 & $\mathrm{N}^{\circ}$ OTRAS NACIONALIDADES \\
\hline V5 & $\mathrm{N}^{\circ}$ MUJERES & V18 & $\begin{array}{l}\text { TASA POBLACION EXTRANJERA. Porcentaje de } \\
\text { población nacida fuera de España. }\end{array}$ \\
\hline V6 & \% POBLACIÓN FEMENINA & V19 & $\begin{array}{l}\text { TASA DE DEPENDENCIA. Porcentaje de población de } \\
\text { más de } 64 \text { años sobre la población de } 16 \text { a } 65 \text { años. }\end{array}$ \\
\hline V7 & \% POBLACIÓN JOVEN & $\mathrm{V} 20$ & $\begin{array}{l}\text { TASA POBLACIÓN INMIGRANTE. Porcentaje de } \\
\text { la población nacida fuera de Baleares }\end{array}$ \\
\hline V8 & No PERSONAS ANCIANAS & V21 & $\begin{array}{l}\text { TASA DE JUVENTUD. Porcentaje de la población } \\
\text { de } 16 \text { a } 29 \text { años sobre la población total }\end{array}$ \\
\hline V9 & $\mathrm{N}^{\circ}$ NACIDOS IB & V22 & $\begin{array}{l}\text { TASA DE ENVEJECIMIENTO. Porcentaje de } \\
\text { la población de más de } 64 \text { años sobre la población total. }\end{array}$ \\
\hline V10 & $\mathrm{N}^{\circ}$ NACIDOS ESPAÑA & V23 & \% Población de más de 64 años. \\
\hline V11 & No NACIDOS EXTRANJERO & V24 & $\begin{array}{l}\text { TASA SOLEDAD ANCIANA. Porcentaje de unidades } \\
\text { de convivencia unipersonal. }\end{array}$ \\
\hline V12 & No COMUNITARIOS & V25 & $\begin{array}{l}\text { VALOR CATASTRAL MEDIO. Valor catastral medio } \\
\text { de los bienes inmuebles del barrio. }\end{array}$ \\
\hline V13 & $\begin{array}{l}\text { No EUROPEOS } \\
\text { NO COMUNITARIOS }\end{array}$ & V26 & $\begin{array}{l}\text { TASA DE ACTIVIDADES ECONÓMICAS. Número } \\
\text { de actividades económicas de alta en Consejería de } \\
\text { Hacienda sobre la población empadronada. }\end{array}$ \\
\hline
\end{tabular}

Fuente: Ayuntamiento de Palma (OMP, 2014). 
Obtenemos un valor único de INSTP para cada barrio en función de su perfil socioeconómico. El método utilizado consiste en la aplicación de un modelo multicriterio ponderal en el que participan un conjunto de variables socioeconómicas a las que se asigna un peso en función de su importancia. El procedimiento se representa en la figura 6 .

El cálculo del INSTP plantea dos problemas: la selección de las variables socioeconómicas que deberán sean representativas del grado de necesidad de transporte público de la población y la asignación de un peso a cada variable que refleje su importancia en la demanda social de transporte público.

Como fuente de información se cuenta con una base de datos socioeconómica de los barrios de Palma construida a partir de datos del Observatorio Municipal de Palma (OMP, 2014) que incluye 26 variables. (tabla 1). Como tarea previa de ayuda a la selección de variables se realiza un análisis factorial con dicha información. Los resultados proporcionan información sobre la correlación entre las variables observadas y permiten simplificar la realidad socioeconómica en un número menor de factores socioeconómicos no observados directamente.

El análisis factorial se ha realizado utilizando el paquete estadístico SPSS versión 21 (@ IBM). Las variables consideradas se relacionan en la tabla 1.

Los resultados del análisis factorial de componentes principales permiten identificar tres factores que acumulan casi el $70 \%$ de la varianza (tabla 2 ):

- El primer factor (F1) puede definirse como Magnitud demográfica, pues aglutina gran parte de las variables relacionadas con la carga poblacional de los barrios (V2, V4, V5, V7, V8, V9, V10) existe una gran correlación entre las mismas. En sí mismas, no representan aspectos de exclusión social o específicos de necesidad social del transporte.

- El segundo factor (F2) representa la Población inmigrante y agrupa aquellas variables relacionadas con población extranjera y no nacida en Baleares (V18, V24 y V26, V20).

- El Envejecimiento y la dependencia definen al tercer factor (F3) que incluye variables relacionadas con el envejecimiento de la población de los barrios (V22, V23). A dicho grupo se añaden la variable que representa la tasa la población femenina (V6). Probablemente este hecho de deba a que la esperanza de vida de las mujeres es más alta y por tanto se relacionan con variables propias de la vejez.

Como resultado del análisis factorial también se observan variables que desarrollan una distribución independiente y están poco correlacionadas con el resto: la tasa de juventud y el valor catastral medio. 
TABLA 2

VARIANZA TOTAL EXPLICADA POR CADA FACTOR Y COEFICIENTES FACTORIALES

\begin{tabular}{l|c|c|c}
\hline \multirow{2}{*}{\multicolumn{1}{c|}{ Factores }} & \multicolumn{3}{c}{ Varianza total explicada } \\
\cline { 2 - 4 } & Total & \% de la varianza & \% acumulado \\
\hline F1. Magnitud demográfica & 11,917 & 45,834 & 45,834 \\
\hline F2. Presencia de inmigración & 3,032 & 11,660 & 57,495 \\
\hline F3. Envejecimiento y dependencia & 2,638 & 10,146 & 67,640 \\
\hline
\end{tabular}

Coeficientes factoriales. Método rotaciónVarimax con normalización Kaiser

\begin{tabular}{c|c|c|c|c|c|c|c}
\hline VARIABLE & F1 & F2 & F3 & VARIABLE & F1 & F2 & F3 \\
\hline V2 &, 988 & & & V16 &, 628 & & \\
\hline V4 &, 988 & & & V3 &, 505 & & \\
\hline V5 &, 986 & & & V25 & & & \\
\hline V8 &, 962 & & & V1 & & & \\
\hline V11 &, 962 & & & V20 & &,- 801 & \\
\hline V14 &, 952 & & & V18 & &, 785 & \\
\hline V7 &, 951 & & & V24 & &, 708 & \\
\hline V10 &, 951 & & & V26 & &, 614 & \\
\hline V9 &, 941 & & & V22 & & &, 876 \\
\hline V13 &, 907 & & & V23 & & &, 859 \\
\hline V15 &, 774 & & & V19 & &,- 534 &, 720 \\
\hline V12 &, 762 &, 357 & & V6 & & &, 659 \\
\hline V17 &, 690 &, 342 & & V21 & & & \\
\hline
\end{tabular}


A partir de estos resultados como se procede a la selección de las variables que serán consideradas para el cálculo del INSTP. El objetivo es que dichas variables representen al máximo las dinámicas sociodemográficas existentes, detectadas a través del análisis multivariante así como otros aspectos que se consideren relevantes. Las variables seleccionadas son las siguientes:

- Población Dependiente (V19, Tasa de Dependencia). Los ancianos y los más jóvenes son usuarios preferentes del autobús ya que no disponen de autonomía para sus desplazamientos, son -por las restricciones que impone su edad- la población más cautiva del transporte público. Esta variable es representativa del tercer factor del análisis factorial ("Envejecimiento y dependencia").

- Población inmigrante (V20, Tasa de población inmigrante). Incluye a la población extranjera perteneciente a colectivos desfavorecidos (latinoamericanos, asiáticos y africanos). Se trata de personas con bajo nivel de renta que normalmente viven en barrios periféricos y precisan de transporte público para sus desplazamientos hacia las zonas de mayor actividad económica. Esta variable representa el segundo factor del análisis factorial ("Presencia de inmigración").

- Población femenina (V6, \% población femenina, Tasa de mujeres). A pesar de las políticas de igualdad desarrolladas en los últimos años, la mujer continúa siendo una usuaria preferente del transporte público. Por ello consideramos necesario seleccionar esta variable de forma preferente. A su está representada por el segundo factor.

- Actividad económica del barrio (V26, Tasa de Actividad Económica). Incorporamos esta variable al entender que el flujo de transporte normalmente se realiza desde zonas residenciales con escasa actividad económica a áreas más céntricas de la ciudad o polígonos de servicios que concentran una mayor actividad económica.

Para poder integrar las variables al análisis multicriterio fueron normalizadas a una escala numérica 0/1. En el caso de la tasa de actividad económica se utilizó el valor inverso, al entender que las áreas pobladas con menor escasa económica muchas veces precisan ver incrementado su nivel de transporte público para no sufrir aislamiento.

La ponderación final de las variables se resuelve mediante el desarrollo de un modelo multicriterio ponderal utilizando el método de las jerarquías analíticas (AHP) (Saaty, 1995). Se realiza un juicio de valor en la comparación de pares de variables asignado un peso para diferenciar la importancia de una variable respecto a la otra. Se ha utilizado la plantilla EXCEL de evaluación AHP propuesta por Klaus Goepel (Goepel, 2013). 


\section{FIGURA 6}

\section{CÁLCULO DEL ÍNDICE DE NECESIDAD SOCIAL DEL TRANSPORTE PÚBLICO (INSTP)}

$$
\operatorname{INSTP}_{(b)}=\sum_{i=1}^{n} W_{(i)} X_{(i)}
$$

i: variable socioeconómica (Población dependiente, Población inmigrante, Población femenina, Actividad económica) $\mathrm{n}$ : número total de variables consideradas (en nuestro caso $\mathrm{n}=4$ )

W: Peso asignado a la variable (Se incorporarán aquí los valores obtenidos como resultado del proceso de asignación de importancia de la valoración AHP).

X: Valor normalizado de la variable según la expresión

Valor normalizado $=\frac{(\text { Valor variable }- \text { Valor mínimo })}{(\text { Valor máximo }- \text { Valor mínimo })}$

La valoración fue realizada por un equipo de seis expertos que asignaron un orden de importancia las variables obteniéndose los resultados expuestos en la tabla 3.

TABLA 3

\section{MATRIZ DE PONDERACIÓN DE CRITERIOS POR PARES Y VECTOR DE PRIORIDAD DE LOS CRITERIOS}

\begin{tabular}{l|c|c|c|c|c}
\hline & & $\begin{array}{c}\text { Tasa de } \\
\text { dependencia }\end{array}$ & $\begin{array}{c}\text { Tasa de } \\
\text { extranjeros }\end{array}$ & $\begin{array}{c}\text { Tasa de } \\
\text { Mujeres }\end{array}$ & $\begin{array}{c}\text { Tasa de } \\
\text { Actividad } \\
\text { Económica }\end{array}$ \\
\hline & & 1 & 2 & 3 & 4 \\
\hline Tasa de Dependencia & 1 & 0 & 2,449 & 3,873 & 4,243 \\
\hline Tasa de Extranjeros & 2 & 0,408 & 0 & 3 & 3,162 \\
\hline Tasa de Mujeres & 3 & 0,258 & 0,333 & 0 & 4,472 \\
\hline Tasa de Actividad Económica & 4 & 0,236 & 0,316 & 0,224 & 0 \\
\hline
\end{tabular}

Eigenvector normalizado y ranked de las variables

\begin{tabular}{l|c|c}
\hline \multicolumn{1}{c|}{ VARIABLE } & Peso & Orden \\
\hline Tasa de Dependencia & $48,8 \%$ & 1 \\
\hline Tasa de Extranjeros & $27,7 \%$ & 2 \\
\hline Tasa de Mujeres & $16,3 \%$ & 3 \\
\hline Tasa de Actividad Económica & $7,2 \%$ & 4 \\
\hline
\end{tabular}


Evaluación de desequilibrios en el análisis de la equidad, a través de la Curva de Lorenz y del coeficiente Gini

A partir de los niveles de servicio de transporte público de cada barrio y los indicadores de demanda (población / INSTP) que hemos obtenido se procede al cálculo de los índices de equidad horizontal y vertical, según las siguientes expresiones:

Equidad Horizontal $=$ Densidad de Población $/$ Nivel de Servicio Equidad Vertical $=$ Índice de Necesidad Social de Transporte Público / Nivel de Servicio

Una mayor equidad horizontal supone un mayor equilibrio entre la población de un barrio y el servicio en transporte público que dispone. A partir del análisis emergerán desequilibrios en barrios densamente poblados y con baja oferta de transporte. Las zonas de menor equidad vertical, por su parte, corresponderán a barrios con elevada necesidad de transporte público y bajo nivel de servicio.

Como instrumento metodológico complementario para la evaluación del nivel de equidad del servicio de transporte hacemos uso de la Curva de Lorenz y del Coeficiente Gini (Delbosc y Currie, 2011; Rodrigue et al., 2013). Ambos se obtienen a partir de la información correspondiente al nivel de servicio, la población y el Îndice Social de Necesidad de Transporte Público para cada uno de los barrios. El coeficiente Gini se obtiene partir del análisis de la proporción de áreas de la curva de Lorenz y se utiliza para la medida de la desigualdad. El valor del coeficiente Gini oscila entre 0 y 1 (figura 7). En nuestro caso un valor 0 correspondería con la distribución perfecta (todos tienen el mismo nivel de servicio de transporte), mientras que un valor 1 supondría una absoluta desigualdad (un barrio, tiene todo el servicio y los demás ninguno).

FIGURA 7

CÁlCULO DEL COEFICIENTE GINI

$$
G=1-\sum_{i=0}^{n}\left(\sigma Y_{i-1}+\sigma Y_{i}\right)+\left(\sigma X_{i-1}+\sigma X_{i}\right)
$$

i: barrio; n: número total de barrios ; $\sigma \mathrm{Y}$ : Porcentaje acumulado de nivel de servicio $\sigma \mathrm{X}$ : Porcentaje acumulado de la población (equidad horizontal) o del INSTP (equidad vertical)

Fuente: Rodriguez et al., 2013

El cálculo de los coeficientes se realizará tanto para el conjunto de los 88 barrios, como para los 73 seleccionados que disponen, como hemos indicado, de una trama urbana más densa. De esa forma se podrá constatar si el nivel de desequilibrio muestra algún tipo de variación en función de la tipología del barrio. 


\section{Análisis del impacto de la modificación de la frecuencia de las líneas de bus en la equidad del servicio}

Finalmente, se analiza el impacto de la modificación de la frecuencia de las líneas sobre la población, para cada uno de los barrios. Para ello, se procede a la generación de escenarios que simulan incrementos en el número de autobuses de paso (frecuencia) y la consecuente disminución de tiempos entre dos buses consecutivos, de cada una de las líneas. Se comparan los valores correspondientes al nivel de servicio, equidad horizontal, equidad vertical y coeficiente Gini, para cada escenario, con los obtenidos en la situación inicial. Este procedimiento permite detectar cuáles son las líneas de autobús urbano que mayor influencia tienen en la dotación de servicio público de transporte en cada uno de los barrios y su influencia en la equidad total del servicio.

\section{RESUlTADOS Y DisCUSIÓN}

Uno de los aspectos más relevantes del trabajo, que puede ser considerado por parte de planificadores y gestores del transporte público en la ciudad de Palma, hace referencia a la distribución territorial de la demanda social de transporte. Los resultados del modelo multicriterio realizado muestran una importante concentración de la demanda de transporte público, expresado en el Índice de Necesidad Social de Transporte Público (INSTP) en los barrios más poblados del ensanche de Palma (Pere Garau, Bons Aires) y en algunas zonas más periféricas y turísticas como Cala Major y Arenal (figura 8). Los primeros tienen un marcado carácter residencial y en ellos se aloja una gran parte de la población inmigrante con escasos recursos, junto a una población local más bien de rentas medias-bajas. Los segundos comparten la función turística -especialmente Cala Major-y residencial, con más de la mitad de población extranjera, población inmigrante que podríamos denominar «residencial» para diferenciarla de la que emigra por razones económicas.

El modelo radio concéntrico de la ciudad configura las infraestructuras viarias y condiciona el trazado de las rutas de transporte público lo cual incide directamente sobre el nivel de servicio proporcionado. La figura 2 muestra la distribución de las líneas de autobús su convergencia en el centro de la ciudad. De hecho, en torno al casco histórico se concentra el 35\% de la oferta del conjunto de líneas. El número de buses por parada dibuja un cinturón de servicio, que corresponde a las avenidas, antiguo trazado de las murallas de la ciudad decimonónica, desde el cual parten una serie de radios que perfilan las principales arterias de entrada y salida de la ciudad (de poniente a levante, a 
través de la calle General Riera, carretera de Valldemossa, o de Sóller, calle Aragón o de Manacor, barrio del Arenal, etc.). En cuanto al nivel de servicio, comprobamos que las paradas del centro pueden llegar a gestionar más de 400 autobuses en un período de 12 horas. Menor dinamismo presentan, sin embargo, las paradas de los ejes principales con niveles que oscilan entre los 100 y 200 servicios de bus en el mismo periodo (figuras 5 y 8).

Este patrón, centralizado tanto en la infraestructura como en el servicio, dificulta la movilidad transversal en la ciudad y causa desequilibrios en el acceso a los equipamientos y a los servicios por parte de su población. El modelo de rutas desplegado prima los desplazamientos periferia-centro-periferia lo cual tendrá consecuencias determinantes en la accesibilidad que, en transporte público y salvo contadas excepciones, pasará forzosamente por el centro de la ciudad. El modelo descrito es asimismo responsable de la configuración de zonas urbanas periféricas, escasamente conectadas con el conjunto de la ciudad, pues cuentan tan sólo, la mayor parte de las veces, con el servicio de una línea de autobús que las une al tejido urbano consolidado de la ciudad.

Los barrios con mayor nivel de servicio son los más céntricos, mientras que los periféricos manifiestan un mayor aislamiento en transporte público (figura 8). Es destacable el incremento de servicio en la zona Sureste de Palma correspondiente a los barrios de Les Meravelles, Can Pastilla y s' Arenal. En este caso, más que las necesidades de la población residente es la actividad turística reflejada en un número elevado de plazas hoteleras, sobre todo en los dos últimos, la que justifica dicho incremento.

El modelo de distribución del servicio tiene una marcada pauta de autocorrelación espacial (índice Moran de 0,45) que nos remite a un modelo agrupado y centralizado. Ello se constata en la elevada correlación entre el número de autobuses de las paradas y su proximidad al centro de la ciudad (plaza de España, por ejemplo), con un coeficiente de correlación que alcanza el 0,7.

Sin embargo, los patrones de distribución de la población, a partir de los del índices de Necesidad Social de Transporte Público y del Nivel de Servicio siguen modelos diferentes y más bien divergentes, cuyo correlato se refleja en la variabilidad de los niveles de equidad en el acceso al servicio de transporte en los distintos barrios (figura 8).

La equidad horizontal presenta un modelo de distribución desequilibrado con importantes diferencias en distintas áreas de la ciudad. Las zonas con nivel de equidad horizontal menor corresponden a sectores con elevada población y un servicio de autobús insuficiente. Al respecto, se distinguen dos zonas principales de déficit que se extienden desde el centro de la ciudad hacia la periferia, una hacia el noroeste y otra hacia el noreste. Se trata fundamentalmente de barrios muy po- 


\section{FIGURA 8}

DISTRIBUCIÓN DE LA DENSIDAD DE POBLACIÓN, ÍNDICE DE NECESIDAD SOCIAL DE TRANSPORTE PÚBLICO, NIVEL DE SERVICIO, Y NIVELES DE EQUIDAD HORIZONTAL Y VERTICAL DEL TRANSPORTE PÚBLICO EN BUS EN LOS BARRIOS MÁS DENSAMENTE POBLADOS DE PALMA

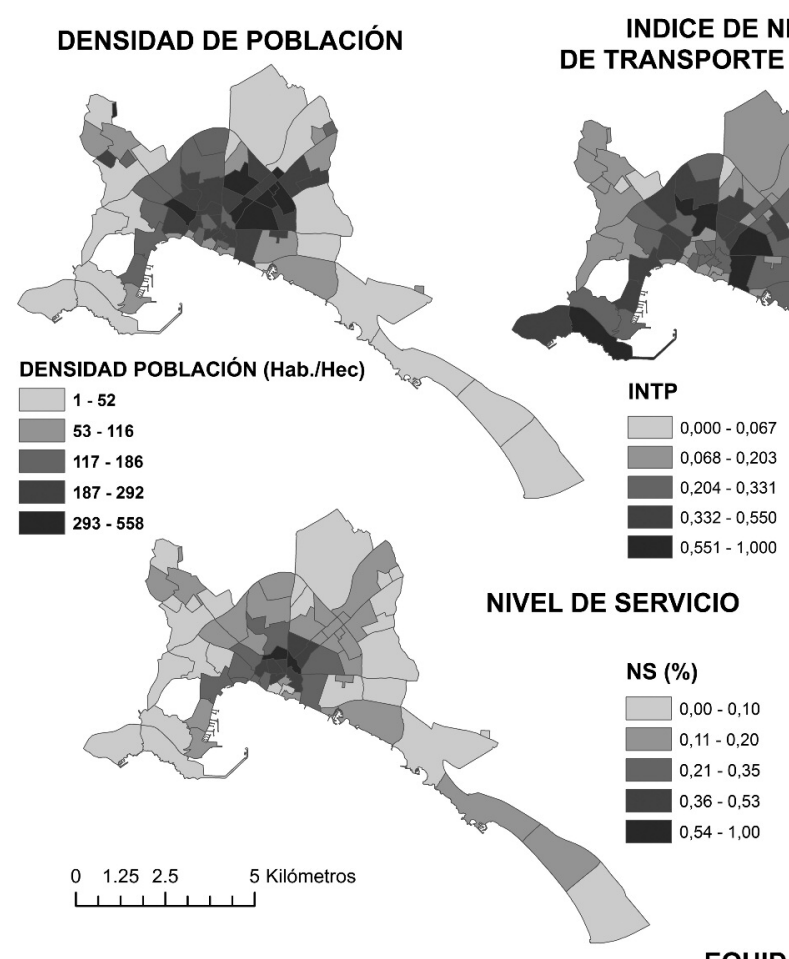

\section{EQUIDAD HORIZONTAL EQUIDAD VERTICAL}

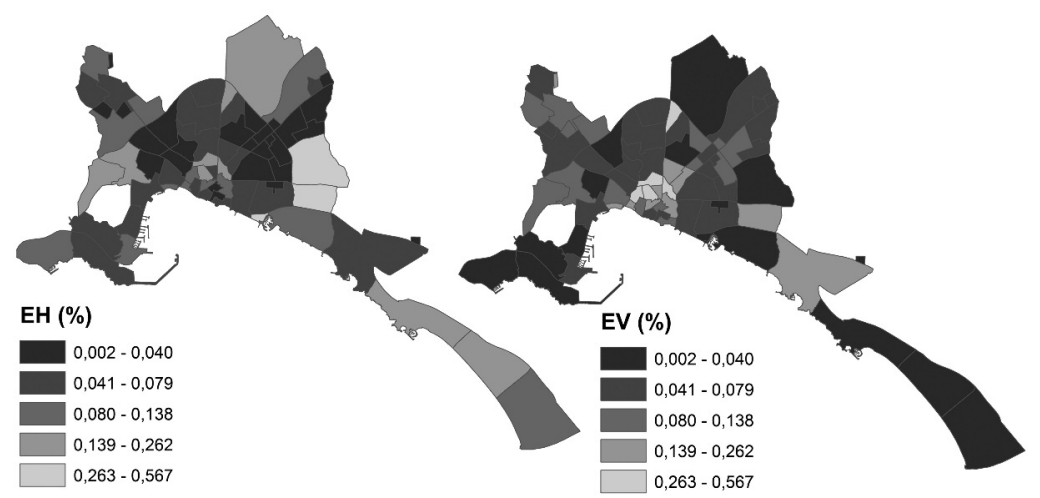


blados del extrarradio (Pere Garau, Son Gotleu, etc.) cuyo servicio de transporte no responde a la densidad de población existente. Los valores más equilibrados de equidad horizontal se dan en la zona céntrica de Palma, en cuyo caso se trata más bien de un exceso de servicio por la elevada concentración de líneas y paradas y la baja densidad demográfica y en barrios de segunda línea, cuya población no es muy elevada y el servicio de bus responde adecuadamente a sus necesidades.

El gráfico de distribución del nivel de servicio de transporte en relación a la población (figura 9) abunda en los comentarios arrojados y permite observar la elevada marginalidad de algunos barrios en cuanto a nivel de servicio y la sobredotación de otros. Las causas principales de este desajuste como veremos más adelante se deben fundamentalmente al trazado de rutas que, como se ha señalado, responde al patrón radio concéntrico de la ciudad.

FIGURA 9

NIVEL DE SERVICIO DE TRANSPORTE PÚBLICO Y POBLACIÓN EN LOS BARRIOS. PALMA

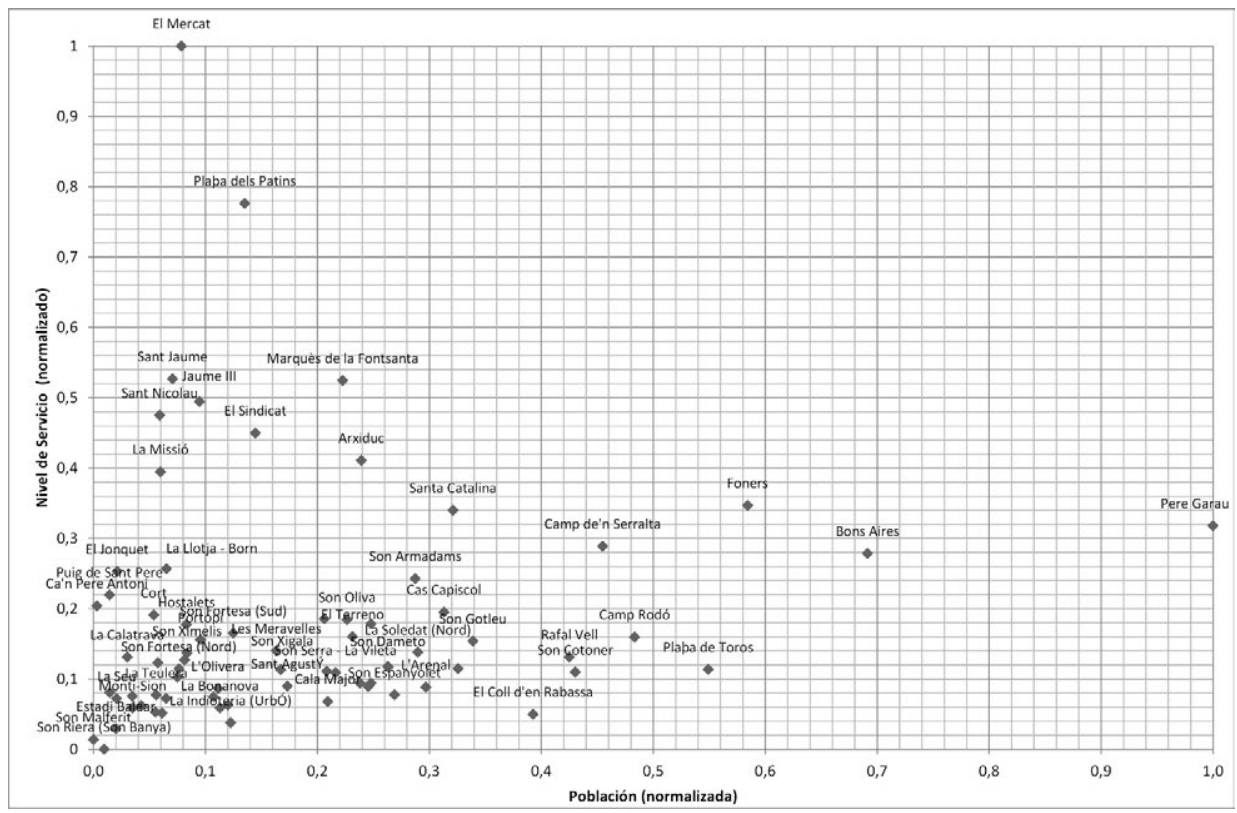

La elevada accesibilidad y el mayor nivel de servicio en el centro de Palma, singularizados principalmente por los barrios Es Mercat y Plaça del Patins, no reflejan un equilibrio real con las demandas de su población residente. En reali- 
dad estas zonas acaparan la recepción de viajes de la periferia, ya que, como añadido al modelo radioconcéntrico, tradicionalmente habían concentrado, y todavía aglutinan, una actividad económica, comercial y de servicios destacada en la ciudad. Sin embargo, en los últimos decenios la dinámica del crecimiento urbanístico ha provocado que gran parte de los grandes equipamientos y servicios colectivos se hayan ido localizando en el extrarradio de la ciudad. Como consecuencia se acusa, por una parte, una falta de servicio en algunas zonas periféricas, tan sólo dotadas de accesibilidad en transporte privado y, por otra, una sobredotación de servicio de transporte público en algunas zonas centrales, como los barrios mencionados. El actual modelo de transporte público favorece que la periferia de Palma aglutine equipamientos, público y privados, y población con escaso nivel de servicio de transporte público. Esta situación repercute gravemente en la sostenibilidad ambiental y económica del servicio público de transporte ya que incentiva el uso del vehículo privado, el único que asegura una movilidad al conjunto de la población y la progresiva obsolescencia del modelo de transporte público. El paradigma de esta situación es la localización de la Universidad de las Illes Balears a $7 \mathrm{Km}$. del centro de la ciudad, los centros Hospitalarios de Son Espases y Son Llátzer, también periféricos, varios centros deportivos o los polígonos industriales y de servicios, localizados más allá del primer cinturón de viales que envuelve la ciudad. En los últimos años se han incluido nuevas líneas transversales o diametrales al servicio que intentan paliar esta situación, pero todavía se está lejos de mejorar el modelo actual.

Si evaluamos la equidad del servicio público utilizando la curva de Lorenz y el coeficiente de GINI, como técnicas estadísticas complementarias, comprobamos como los desequilibrios en la ciudad en la dotación de transporte público son notables. Las curvas presentadas en la figura 9 muestran un alejamiento considerable de la situación de equilibrio, tanto cuando se considera propiamente la población residente como cuando se analiza la necesidad social de transporte público.

Los resultados del coeficiente GINI muestran un mayor nivel de desequilibrio de la equidad vertical $(0,386)$, expresada a partir del Índice de Necesidad Social del Transporte público y el nivel de servicio, frente a la horizontal $(0,345)$ que refleja más bien la relación entre la densidad demográfica y el servicio de autobús. La magnitud de estos indicadores es moderadamente baja por lo que su resultado debe de interpretarse de forma menos negativa. Aun así, los índices pueden interpretarse como una falta de adaptación del servicio de transporte público a las necesidades de su población, tanto por su cuantía, como por sus características socioeconómicas, que incide en la desigualdad social de la ciudad en el acceso a la movilidad en medios públicos. Sin em- 
FIGURA 10

CURVAS DE LORENZ Y COEFICIENTES GINI DEL SERVICIO DE TRANSPORTE EN AUTOBÚS EN RELACIÓN A LA POBLACIÓN Y AL ÍNDICE DE NECESIDAD DE TRANSPORTE PÚBLICO. PALMA

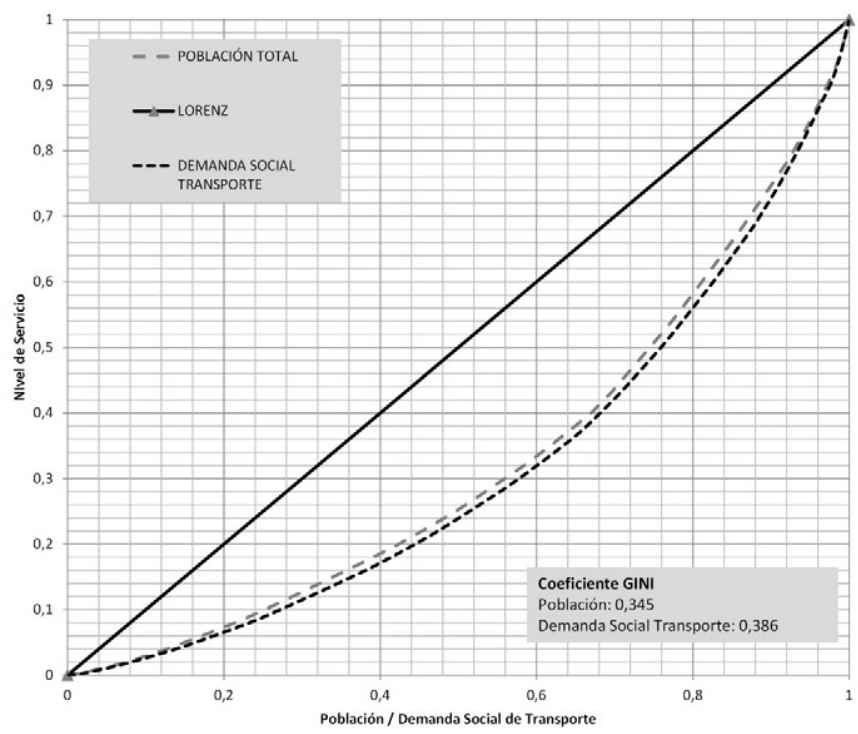

bargo, si realizamos el cálculo de los coeficientes para el total de los 88 barrios de la ciudad, en lugar de las 73 unidades de análisis que hemos utilizado para nuestro estudio, obtenemos unos valores sensiblemente superiores: 0,405 para la población y 0,448 para la demanda social de transporte. Ello supone mayores niveles de desequilibrio globales en la ciudad, es decir, la inclusión de los barrios con población más bien dispersa provoca mayores desajustes entre el servicio de transporte y la población servida, tanto por su cuantía como por sus características socioeconómicas.

El análisis del impacto (sensibilidad) de la modificación de la frecuencia de las líneas de autobuses demuestra que no todas ellas desempeñan el mismo rol en la dotación de servicio a los barrios (figura 11).

Entre las líneas con mayor sensibilidad en el servicio global de la ciudad destacan la L7, L8, L5 y L15 (figura 2). Se trata de líneas con rutas de largo recorrido, cuyas frecuencias son mayoritariamente altas, es decir el tiempo de espera entre dos autobuses consecutivos es bajo, conectan barrios periféricos y cruzan por el centro la ciudad. Una pequeña variación en la frecuencia de estas líneas tiene un efecto muy notable sobre el servicio general. 
FIGURA 11

IMPACTO (SENSIBILIDAD) DE LA MODIFICACIÓN DE LA FRECUENCIA (INCREMENTO DE 10 MINUTOS) DE LAS LÍNEAS DE TRANSPORTE EN AUTOBÚS EN LA EQUIDAD HORIZONTAL Y VERTICAL EN EL CONJUNTO DE LA CIUDAD. PALMA

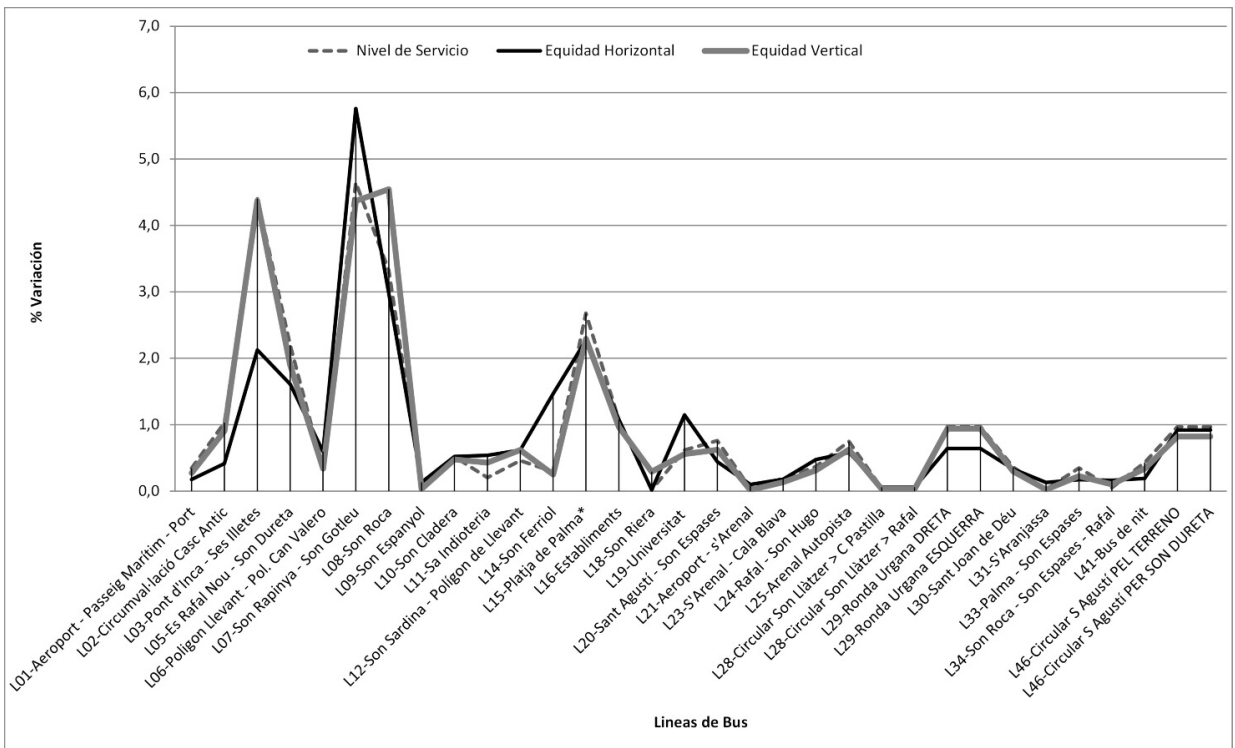

La modificación de la frecuencia de las líneas impacta tanto en la equidad horizontal como en la vertical. En la primera destacan las líneas L7, L15 y L3 y el aumento del nivel de servicio reflejado en un mayor número de buses tiene un efecto directo sobre la población servida. Por otro lado, las líneas L8, L7 y L3, una de las pocas transversales con las que cuenta la ciudad, destacan por su papel en la equidad vertical. Es decir, actuaciones sobre estas líneas provocarían efectos directos sobre colectivos con elevada dependencia y alta necesidad potencial del transporte público.

En el otro extremo se configuran un conjunto de líneas con exceso de servicio y que realizan recorridos en zonas turísticas (L15, L25 y L46). Finalmente, las líneas L28, L31 y L34, dos de ellas circulares, tienen escasa representatividad en el volumen de desplazamientos globales de la ciudad pero proporcionan accesibilidad a barrios aislados del continuum urbano y con escaso poblamiento.

El impacto de las frecuencias se pone igualmente de manifiesto al constatar una estrecha correlación, en este caso inversa, entre el nivel de servicio y la fre- 
cuencia horaria (Rho de Spearman: -0,856), es decir, que los barrios que acusan un índice de servicio mayor son aquellos en los que los tiempos de espera entre dos autobuses consecutivos son menores, es decir, las líneas cuentan con mayores frecuencias. La gestión de las frecuencias es, por tanto, un instrumento de gran utilidad para asegurar la equidad del sistema de transporte. El efecto de las frecuencias y el recorrido de las líneas son las claves para dotar de mayor relevancia a una línea u otra y, por tanto, gestionar el nivel de servicio y equidad a las distintas zonas de la ciudad. El cambio de recorridos de las rutas tiene un efecto más directo sobre el nivel de servicio, por lo que sus repercusiones políticas y, sobre todo las económicas y sociales, son mucho más destacadas.

Si analizamos el servicio de transporte ofertado en los principales barrios de la ciudad de Palma observamos la gran dependencia funcional respecto de determinadas líneas de transporte público en algunos de ellos (figura 11). El barrio de Pere Garau, como ejemplo, tiene una gran dependencia funcional de las líneas L5, L7, L24 y L29. Con ello se indica que un incremento su frecuencia horaria produciría una mejora considerable del servicio. Algo similar pasa en el barrio de Camp Rodó, el otro ejemplo escogido, en el que las líneas clave, en este caso, son las L16, L29 y L33. Si se realiza este ejercicio de simulación para los distintos barrios de la ciudad es posible identificar con precisión el papel que juega cada línea en cada uno de los barrios, es decir, el nivel de sensibilidad aportado por cada línea en cada zona o el impacto que cada una de ellas genera, al modificar sus frecuencias. Consecuentemente es posible argumentar un modelo de redistribución horaria para que el sistema de transporte público urbano aporte más equidad a la ciudad, en este caso a la ciudad de Palma.

FIGURA 12

IMPACTO (SENSIBILIDAD) DE LA MODIFICACIÓN DE LA FRECUENCIA (*) DE LAS LÍNEAS DE TRANSPORTE EN AUTOBÚS EN LOS BARRIOS DE PERE GARAU Y CAMP RODÓ. PALMA
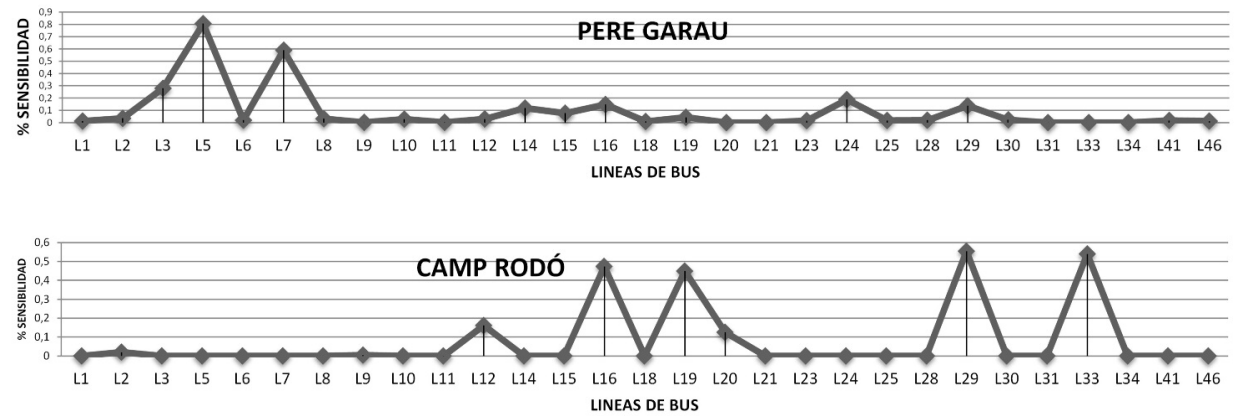

Estudios Geográficos, Vol. LXXVII, 281, pp. 619-646, julio-diciembre 2016 ISSN: 0014-1496, eISSN: 1988-8546, doi: 10.3989/estgeogr.201621 


\section{CONCLUSIONES}

Palma constituye un ejemplo de ciudad en la que la red actual del servicio de autobuses plantea una serie de problemas, muchas veces comunes en las ciudades históricas, por haberse desarrollado orgánicamente a remolque de las demandas generadas por el crecimiento de nuevas zonas urbanizadas y de equipamientos en su periferia. Los desajustes y disfunciones, en la mayor parte de ciudades occidentales, aunque aquí no se hayan analizado, son múltiples y variados: falta de accesibilidad entre barrios, ausencia de sincronismo entre rutas que favorezca los transbordos, redundancia con otros sistemas de transporte público, la estructura geométrica de la red, la baja velocidad comercial, etc. Ese conjunto de factores, a los que debemos sumar, para la ciudad de Palma, una morfología radioconcéntrica que se refleja en el trazado y recorrido de las líneas de autobús, provoca una falta de equidad en el servicio que aconseja un replanteamiento estructural del sistema de transporte público y de su red, que incluya diversas tareas, como: el diseño de rutas y paradas en función de un mayor o menor crecimiento demográfico y urbanístico y de la implementación de nuevos equipamientos; el establecimiento de frecuencias adaptadas a las necesidades; la adaptación de las infraestructuras a la demanda de servicio o la propia coordinación semafórica, que facilite el tráfico del transporte público, entre otras. Dado que no es fácil asumir los costes y riesgos asociados a cambios estructurales en los modelos de transporte, tanto a escala urbana como a otras escalas de análisis, es aconsejable disponer de metodologías que favorezcan el diagnóstico dinámico de la red y que, mediante pequeños ajustes, faciliten la adaptación del servicio a los nuevos requerimientos de la población.

La metodología propuesta constituye un avance en la integración de diversas técnicas de análisis de datos geográficos y socioeconómicos para el estudio de la equidad del transporte público. En especial, el análisis del impacto (sensibilidad) de las frecuencias en las rutas de transporte y la optimización de horarios puede mejorar de forma notable la adaptación de un servicio de transporte a un ámbito geográfico determinado. Desde esta perspectiva, el presente estudio plantea un método sencillo de diagnóstico de la equidad del servicio de autobús urbano y propone la posibilidad de ajuste de las frecuencias a fin de adaptar el servicio a las necesidades reales, sobrevenidas por las densidades demográficas y por las propias características socioeconómicas de la población, en constante mutación.

Los sistemas de información geográfica, en combinación con algunas técnicas estadísticas utilizadas en este trabajo, constituyen un instrumento meto- 
dológico adecuado para el análisis y el seguimiento de la equidad del transporte público. Ayudan a elaborar un diagnóstico certero y, a su vez, permiten la simulación de escenarios para evaluar los efectos territoriales y sociales de posibles actuaciones futuras derivadas de los cambios en las dinámicas urbanas.

La ciudad de Palma presenta desequilibrios moderados en la equidad del transporte público de autobús, el único modo público extendido a lo largo de la ciudad. Se manifiesta una gran dependencia del modelo de las líneas de largo trazado, tanto las urbanas como las interurbanas, algunas de ellas transversales, que unen barrios periféricos distantes con el nodo central, el centro histórico de la ciudad. El modelo más bien centralizado supone una pérdida notable de equidad en barrios demográficamente relevantes, pero alejados del centro, cuya población, más bien de escasos recursos, engloba un porcentaje considerable de colectivos marginales (extranjeros, ancianos, etc.). Por ello, sería deseable revisar el actual modelo de servicio de la red y adaptarlo a las necesidades sociales de los barrios a los que sirve. En primer lugar, mediante un ajuste en las frecuencias pero, progresivamente, redefiniendo el modelo de rutas y paradas, a fin de dotar de mayor accesibilidad a las zonas de la periferia y poder mejor satisfacer las necesidades de movilidad de sus habitantes.

Finalmente, cabe añadir que los modelos de sostenibilidad del transporte se estructuran en base a tres componentes: movilidad observada, transporte público y urbanismo. La hipótesis establecida es que los patrones de movilidad y la estructura de la red de transporte público guardan una clara correspondencia con el modelo territorial de la ciudad (Diaz et al., 2007) por ello una reestructuración del sistema de transporte debe ir coordinada tanto con el modelo urbanístico como con la propia dinámica económica del territorio. Con todo, y aún sin producirse grandes cambios, los ajustes del sistema pueden contribuir a mejorar la calidad de vida de muchos de los usuarios del transporte público y constituyen un eslabón más en la construcción de una ciudad más equitativa y sostenible.

\section{Agradecimientos}

El trabajo se ha desarrollado en el marco del proyecto CIVITAS Dyn@mo. "DYNamic citizens @active for sustainable MObility" n 296057, Seventh Framework Programme, UE (2013-2016). Se agradece el apoyo de la Empresa Municipal de Transportes de Palma y del Departamento de Movilidad del Ayuntamiento de Palma en el suministro de información. 


\section{REFERENCIAS}

Agència de Turisme de les Illes Balears (2013): El turisme a les Illes Balears. Anuari 2013, Palma, Conselleria de Turisme i Esports, Govern de les Illes Balears.

Ajuntament de Palma (2013): PGOU. Revisió del Pla General d'Ordenació Urbana de Palma de Mallorca, Memòria d'Informació i Anàlisi 06. Inédito

Ajuntament de Palma (2014): Pla de Mobilitat Urbana Sostenible (PMUS), documento Inédito.

Biba, S., Curtin, K. M. y Manca, G. (2010): "A new method for determining the population with walking access to transit", Int. J. Geogr. Inf. Sci, 24/3, pp. 347-364.

Casas, I. (2007): "Social Exclusion and the Disabled: An Accessibility Approach", The Professional Geographer, 59/4, pp. 463-477.

Cipriani, E., Gori, S. y Petrelli, M. (2012): "Transit network design: A procedure and an application to a large urban area", Transp. Res. Part C Emerg. Technol, 20/1, pp. 3-14.

Cooco, R. G. y Silveira, M. R. (2011): "Transporte público coletivo: acessibilidades e crise nas cidades do interior paulista", in M. R. Silveira (org.), Circulaçao, transportes e logística. Diferentes perspectivas, Sao Paulo, Outras Expressoes.

Currie, G. (2010): "Quantifying spatial gaps in public transport supply based on social needs", J. Transp. Geogr., 18/1, pp. 31-41.

Delbosc, A. y Currie, G. (2011): "Using Lorenz curves to assess public transport equity”, J. Transp. Geogr., 19/6, pp. 1252-1259.

Diaz, M., Cantergiani, C., Salado, M., Rojas, C. y Gutierrez, S. (2007): "Propuesta de un Sistema de Indicadores de Sostenibilidad para la movilidad y el transporte urbanos. Aplicacion mediante SIG a la ciudad de Alcalá de Henares", Cuad. de Geogr., 81-82, pp. 31-50.

Empresa Municipal de Transportes de Palma (EMT) (2014): http://www.emtpalma.es, consulta 10/11/2014.

Farahani, R. Z., Miandoabchi, E., Szeto, W. Y. y Rashidi, H. (2013): “A review of urban transportation network design problems”, Eur. J. Oper. Res., 229/2, pp. 281-302.

Garcia-Palomares, J. C., Gutierrez, J. y Cardozo, O. D. (2013). "Walking accessibility to public transport: an analysis based on microdata and GIS", Environ. Plan. B Plan. Des., 40/6, pp. 1.087-1.102.

Goepel, K. D. (2013): "Implementing the Analytic Hierarchy Process as a Standard Method for Multi-Criteria Decision Making In Corporate Enterprises - A New AHP Excel Template with Multiple Inputs", Proc. Int. Symp. Anal. Hierarchy Process, 1-10: http://bpmsg.com/new-ahp-excel-template-with-multiple-inputs/ Consulta: Septiembre 2014. Verificada: 10/12/2015.

Gutiérrez, J. y García-Palomares, J. C. (2008): "Distance-measure impacts on the calculation of transport service areas using GIS", Environ. Plan. B Plan. Des., 35/3, pp. 480-503.

Institut d'Estadística de les Illes Balears (IBESTAT) (2014): http://ibestat.caib.es, consulta: 10/11/2014. 
Instituto Geográfico Nacional (IGN) (2014): Cartociudad, http://cartociudad.es/ portal, consulta: 10/11/2014.

Hernández, D., y Witter, R. (2011): "Entre la ingeniería y la antropología: hacia un sistema de indicadores integrado sobre transporte público y movilidad", Revista transporte y territorio, 4, pp. 29-46.

Huang, Z. (2003): Data Integration For Urban Transport Planning, Utrech, University.

Litman, T. (2002): "Evaluating Transportation Equity", World Transp. Policy Pract, 8/2.

Litman, T. (2014): Evaluating Public Transit Benefits and Costs, Victoria, Canadá.

Moreno, A. y Prieto, E. (2004): "Evaluación de procedimientos para delimitar áreas de servicio de líneas de transporte urbano con sistemas de información geográfica", Investig. Reg., 2, pp. 85-102.

Observatorio Municipal de Palma (OMP)(2014): http://www.observatori palma.org, consulta 10/11/2014.

Ortúzar, J. de D. y Román, C. (2003): "El problema de modelación de demanda desde una perspectiva desagregada: el caso del transporte", EURE2, XXIX/88, pp. 149171.

Pedersen, O. W. (2010): "Environmental Principles and Environmental Justice", Environmental Law Review, 12/1, pp. 26-49, doi.org/10.2139/ssrn.1592850.

Rodrigue, J. P., Comtois, C. y Slack, B. (2013): The geography of transport systems. Routledge:https://people.hofstra.edu/geotrans/eng/methods/ch4mlen.html, consulta: Septiembre 2014. Verificada: 10/12/2015.

Saaty, T. L. (1995): "Transport Planning with Multiple Criteria - the Analytic Hierarchy Process Applications and Progress Review", J. Adv. Transp., 29/1, pp. 81-126.

Souche, S. 2010: "Measuring the structural determinants of urban travel demand", Trans Policy, 17/3, pp. 127-134.

Fecha de recepción: 30 de enero de 2015.

Fecha de aceptación: 2 de marzo de 2015. 